\title{
Uso de buenas prácticas en la gestión de proyectos de TI
}

\section{Use of good practices in ti project management}

María Elizabeth Arteaga García

ESAI Business School de la Universidad Espíritu Santo, Ecuador

Mayra Rosibel Pazmiño Castro

ESAI Business School de la Universidad Espíritu Santo, Ecuador

Autor para correspondencia: marteagag@uees.edu.ec, mayra.pazmino@latam.com Fecha de recepción: 25 de Agosto 2017 - Fecha de aceptación: 15 de Enero de 2018

Resumen: Este artículo tiene por objeto identificar la aplicación de buenas prácticas para la gestión de proyectos en organizaciones TI del Ecuador, y así explorar el grado de madurez que poseen esta clase de organizaciones, para lo cual se seleccionaron tres empresas: LATAM Ecuador, Jarflex y Grupo Link, cuyos líderes de proyecto se les envió un cuestionario digital, donde señalaron información de su organización y de su proyecto más relevante. Se encontró que aunque dos de las organizaciones utilizan las buenas prácticas del PMI® y la metodología waterfall, sus calificaciones de autoevaluación de éxito en la gestión de proyectos difieren notablemente, y los demás estándares internacionales y directrices (IPMA, PRINCE2, ISO, entre otros) son desconocidos, muestra del bajo grado de madurez de las organizaciones TI ecuatorianas en la gestión de sus proyectos.

Palabras clave: gestión de proyectos; TI; estándares

Abstract: This article aims to identify the application of good practices for project management in TI organizations in Ecuador, and thus explore the degree of maturity that this class of organizations possess, for which three companies were selected: LATAM Ecuador, Jarflex and Grupo Link, whose project leaders were sent a digital questionnaire, where they indicated information about their organization and their most relevant project. It was found that although two of the organizations use PMI® good practices and the waterfall methodology, their selfassessment ratings for project management success differ significantly, and the other international standards and guidelines (IPMA, PRINCE2, ISO, among others). ) are unknown, showing the low degree of maturity of Ecuadorian TI organizations in the management of their projects.

Key words: project management; TI; standards 


\section{Introducción}

Un proyecto se define como un trabajo no repetitivo que requiere planificación y la definición de objetivos, costos y tiempo (Escandón, 2005), y por lo tanto, es clave una gestión apropiada que implique la aplicación de conocimientos, habilidades, herramientas y técnicas para satisfacer los requisitos solicitados (PMI, 2013). Un proyecto tecnológico requiere a más de la comprensión de la dimensión del negocio, la comprensión de la dimensión tecnológica (Fuster, Hormigo, Joana, \& Rodríguez, 2011). De acuerdo a un estudio realizado por The Standish Group International (2013), las cifras que se manejan respecto a la gestión de proyectos de Tecnologías de la Información (TI) no son muy alentadoras, $18 \%$ fallan rotundamente y $49 \%$ solo termina si es intervenido, y adicionalmente, el $32 \%$ de los empleados trabaja con información incompleta, sin compartirla con sus proveedores o clientes. Salinas Duarte (2007) puntualiza elementos repetitivos que causan el fracaso de los proyectos TI, relacionados mayormente con problemas de gestión que técnicos.

Este artículo tiene por objeto identificar la aplicación de buenas prácticas para la gestión de proyectos en organizaciones TI del Ecuador, y así explorar el grado de madurez que poseen esta clase de organizaciones en lo que respecta a la dirección de proyectos. Para los efectos se seleccionaron tres empresas: LATAM Airlines Ecuador, Jarflex - Desarrollo de Soluciones Informáticas y Grupo Link. A continuación se presenta el desarrollo de la investigación, sus resultados y conclusiones.

\section{Marco Teórico}

\section{Historia de la Gestión de Proyectos}

De acuerdo a Weaver (2012), la gestión de proyectos data de 1896, cuando un economista polaco, ingeniero e investigador Karol Ademiecki presenta su técnica "Harmonograma", donde se ubican actividades, representadas por barras verticales, con sus predecesoras, sucesoras, y su respectiva duración. En 1910, Henry Laurence Gantt dio a conocer el "Diagrama de Gantt", facilitando un mejor control y planificación (Vilar, 2013). En 1916, Henry Fayol, pionero de la doctrina administrativa, describe la administración como un proceso que requiere: planear, organizar, dirigir, coordinar y controlar (Zapata, Murillo, y Martínez, 2006). A finales de los 50`s, en Estados Unidos se crean las metodologías para planificación y monitoreo de proyectos: CPM (Critical Path Method), PERT (Program Evaluation and Review Technique), y PDM (Precedence Diagramming Method), conocida también como la Actividad en Nodo (Stretton, 2007). Posteriormente, en 1962, se creó la WBS (Work Breakdown Structure), para el manejo del alcance de un proyecto (Haugan, 2002). Como consecuencia del nacimiento de las herramientas CPM y PERT, se gestaron eventos de análisis e intercambio de conocimientos por parte de expertos, convirtiéndose en asociaciones de profesionales vinculados a la gestión de proyectos,en 1967 se fundó IPMA® (International Project Management Management Association) en Europa, y en 1969, nace el PMI® ( Project Management Institute) en Estados Unidos. IPMA® y PMI® son las más antiguas asociaciones de profesionales en gestión de proyectos. 


\section{Gestión de Proyectos TI}

Los proyectos TI son aquellos que comprenden la configuración de infraestructura TI (hardware, software, y networking), en todos los departamentos de una organización, con el objeto de manejar sus procedimientos de procesamiento de información (Chemuturi, 2013). The Standish Group International (2013), señala que de cada 100 proyectos de TI, 39 son exitosos (se cumple la triple restricción planificada de alcance, tiempo y costo), 43 culminan sin ser exitosos (no se cumple total o parcialmente la triple restricción planificada), y 18 son cancelados antes de su culminación y no lograron cumplir con los entregables. Kappelman, McKeeman, y Zhang (2006), identificaron las principales 12 señales tempranas de alerta de fracaso de proyectos TI (Ver tabla 1), las cuales si son gestionadas a tiempo, incrementa las probabilidades de contar con un proyecto exitoso.

Tabla 1.

\begin{tabular}{ll}
\hline Riesgos relacionados con la gente & Riesgos relacionados con los procesos \\
\hline Falta de compromiso de altos ejecutivos & Falta de documentación de los requisitos y \\
& sus factores de éxito \\
Director de Proyecto débil & No existe un proceso de control de cambios \\
Falta de compromiso de los interesados & Planificación/gestión de cronograma poco \\
& efectiva \\
Falta de compromiso del equipo de trabajo & Ruptura de comunicación entre \\
& involucrados \\
Los miembros del equipo carecen de conocimiento sobre & Los recursos son asignados a proyectos de \\
los requisitos y/o habilidades de trabajo & mayor prioridad \\
Los expertos están sobreasignados de actividades & No hay el caso de negocio que sustente el \\
& proyecto \\
\hline
\end{tabular}

Fuente: Kappelman, McKeeman, y Zhang (2006)

La aplicación de buenas prácticas en la gestión de proyectos favorece a que estos culminen con éxito, de allí sus ventajas: Genera una práctica estandarizada de gestión de proyectos en la organización, mejora las comunicaciones entre los interesados del proyecto, favorece al trabajo en equipo, facilita el proceso de control y monitoreo, y genera ahorros de tiempo y costos, sin embargo, es el director de proyectos quien define cuáles métodos son los más apropiados según la situación específica que enfrente (Ilies, Crisan, y Muresan, 2010).

\section{Estándares internacionales y directrices en la Gestión de Proyectos}

Las buenas prácticas se han generado como parte de la evolución de los negocios y la aplicación de la gestión de proyectos a nivel global, incluyen directrices y estándares internacionales, construidos a partir de la experiencia de expertos, con el propósito de mejorar las probabilidades de éxito en el logro de los objetivos de un proyecto (Ilies, Crisan, y Muresan, 2010). Los principales estándares internacionales y metodologías en gestión de proyectos se presentan en la Tabla 2.

Tabla 2. Estándares internacionales y directrices en gestión de proyectos

\begin{tabular}{cc}
\hline $\begin{array}{c}\text { Estándares } \\
\text { Internacionales/Directrices }\end{array}$ & Descripción \\
\hline $\begin{array}{c}\text { Project Management Body of } \\
\text { Knowledge }(\boldsymbol{P M B O K})\end{array}$ & $\begin{array}{c}\text { Es una guía, elaborada por el PMI }{ }^{\circledR}, \text { que contiene buenas prácticas, } \\
\text { herramientas y técnicas que se aplican para la mejora de la gestión de los }\end{array}$ \\
\hline
\end{tabular}




\section{IPMA Competence Baseline (IPMA ICB®)}

\section{Project in Controlled Enviroments (PRINCE2)}

\section{Estándares \\ Internacionales/Directrices ISO 21500:2012}

\section{Guidebook for Project and \\ Programa Management for Enterprise Innovation (P2M)}

proyectos. Su primera edición oficial fue publicada en 1996, la segunda en el 2000, la tercera en el 2004, la cuarta en el 2008 y se encuentra vigente su quinta edición, publicada en el 2013. Contiene 47 procesos, que se agrupan en 10 áreas de conocimiento y 5 grupos de procesos. Las áreas de conocimiento son: Integración, Alcance, Tiempo, Costos, Calidad, Recursos Humanos, Comunicaciones, Riesgos, Adquisiciones e Interesados. Los grupos de procesos son: Inicio, Planificación, Ejecución, Monitoreo y Control, y Cierre.

Es un estándar, elaborado por IPMA, que combina teoría, prácticas y demandas sobre gestión de proyectos de 60 asociaciones miembros. Es utilizada por individuos interesados en mejorar sus competencias en gestión de proyectos, programas y portafolios, así como candidatos y asesores interesados en la certificación. Es simbolizada por el "ojo de la competencia", que representa todos los elementos de la gestión de proyectos que deben ser visualizados por el director de proyectos cuando evalúa una situación. La línea base de la competencia comprende 46 elementos: 20 relacionados con técnicas de gestión de proyectos, 15 con el comportamiento profesional del personal de gestión de proyectos, y 11 con el contexto del proyecto.

Es un método efectivo para la gestión de proyectos, elaborado por the British Office of Government Commerce, utilizado ampliamente por el Gobierno de Reino Unido y el sector privado. PRINCE fue creado en 1989, y en 1996 fue publicado el PRINCE2, a partir de la contribución de un consorcio de 150 organizaciones europeas. Ha sido actualizado en los años 2002, 2005 y 2009. Las principales características de PRINCE 2 son: Enfoque en la justificación del negocio, definición de una estructura organizacional para el equipo de gestión del proyecto, planificación basada en el producto, énfasis en dividir el proyecto en etapas manejables y controlables, y flexibilidad que puede ser aplicada en el nivel apropiado del proyecto.El estándar PRINCE 2 señala qué, cuándo y cómo se debe hacer en el proyecto, y por quién.

\section{Descripción}

Este estándar, elaborado por el International Organization for Standardization (ISO), orienta sin profundizar en detalles, sobre conceptos y procesos relacionados con la gestión de proyectos, sean públicos o privados, independientemente de su tamaño, complejidad y duración. Hace referencia a 40 procesos, que pueden ser visualizados desde dos perspectivas: por procesos o por temas. Son 5 grupos de procesos: Inicio, Planificación, Implementación, Control y Cierre, y son 10 temas: Integración, Involucrado, Alcance, Recursos, Tiempo, Costo, Riesgos, Calidad, Adquisiciones y Comunicaciones Entre los estándares ISO 21500:2012 y PMBOK® hay muchas similitudes, pero también existen diferencias.

Estándar desarrollado por Engineering Advancement Association of Japan (ENNA) con el financiamiento del gobierno Japonés, y culminado en el 2002, que se caracteriza por: la combinación de guías de la gestión de proyectos y programas y su orientación a la misión, al mejorar el valor del negocio tomando en consideración los cambios del entorno y facilitando el hallazgo de soluciones para retos complejos. De acuerdo al Project Management Association of Japan - PMAJ (2017) su estructura sigue el orden de la Norma ISO 21500:2012. Esta guía considera tres tipos de proyectos: desarrollo de conceptos (scheme model), 
implementación (system model) y operación (service model), conocido este enfoque como " $3 \mathrm{~S}$ ".

Fuentes: (PMI, 2017; IPMA, 2017; PRINCE2, 2017; ISO, 2017; PMAJ, 2017; Drob y Zichil, 2013).

\section{Metodología}

Para esta investigación se seleccionaron tres organizaciones TI del Ecuador: LATAM Airlines Ecuador, Jarflex - Desarrollo de Soluciones Informáticas y Grupo Link. Una vez identificado el líder de proyecto, vía correo electrónico, se le remitió el link del cuestionario (elaborado en Google Forms), el cual constó de quince preguntas distribuidas en dos secciones: 1) Información de la organización, y 2) Evaluación de la gestión de su proyecto más relevante, en lo concerniente a: alcance, costo, tiempo, calidad, riesgo y calidad), empleando escala Likert de cinco puntos, y el uso de buenas prácticas. Para el análisis estadístico descriptivo de los datos se utilizó el software SPSS. En la Tabla 3 se presenta una breve descripción de cada una de las empresas seleccionadas para el estudio.

Tabla 3. Empresas TI: Breve descripción

\begin{tabular}{ccl}
\hline Empresa & $\begin{array}{c}\text { Cargo del } \\
\text { entrevistado }\end{array}$ & Descripción \\
\hline LATAM & IT Manager & LAN Ecuador® (Líneas Aéreas Nacionales del Ecuador) nace en junio \\
Airlines Ecuador & & de 2002 a partir de una alianza comercial con la Empresa Ecuatoriana de \\
& Aviación. En el año 2008, Aerolane logra la apertura del Gobierno del \\
& Ecuador para explotar rutas nacionales A partir de 2012, la matriz chilena \\
& del grupo formalizó un acuerdo de fusión con la más grande de las líneas \\
& aéreas de Brasil: TAM Airlines, formando así la empresa que hoy opera \\
& en los diferentes países con la marca única: LATAM. Entre el 2012 y el \\
& 2015, LATAM Ecuador ${ }^{\circledR}$ transportó más de dos millones de pasajeros. \\
& Actualmente la operación en Ecuador tiene su matriz en Guayaquil, y \\
& cuenta con más de 1000 colaboradores.
\end{tabular}

\begin{tabular}{lr}
\multicolumn{1}{c}{ Jarflex - } & Gerente \\
Desarrollo de & General \\
Soluciones & \\
Informáticas &
\end{tabular}

Empresa

Grupo Link

\section{Cargo del entrevistado}

Director Comercial
Jarflex S.A. nace en el año 2009, es una empresa dedicada a desarrollar soluciones informáticas y empresariales con tecnología de punta, caracterizándose por su excelente planificación, desarrollo, implementación y mantenimiento, creando un marco de trabajo flexible para los clientes y un ambiente creativo e innovador para sus colaboradores. Comienza su actividad en el área de desarrollo y soporte de sitios Web, luego incursiona en el desarrollo de proyectos tecnológicos del sector inmobiliario, y más tarde en el área de levantamiento de información y desarrollo de aplicaciones administrativas y tecnológicas en el sector aeronáutico. Entre el 2009 y el 2016, Jarflex S.A. ha trabajado en proyectos nacionales e internacionales, incrementando así su experiencia en el campo.

\section{Descripción}

Grupo - Link nace como Dayscript en el año 2006 en Colombia, desarrollando productos digitales web para proyectos de operadoras móviles, y en el año 2009, empieza operaciones en el Ecuador. Entre el 2010 y el 2014, experimentó un gran crecimiento tanto en su portafolio de clientes como proyectos y capacidad productiva. En el 2015, a partir de la inversión de capital de varios países de Latinoamérica, se fusionan sus empresas hermanas y se consolida el Grupo Link. Al día de hoy, Grupo 
Link, ofrece servicios en: desarrollo web, tradicional y aplicaciones móviles,integración móvil, marketing digital, marketing deportivo, asesoría estratégica y call center. Cuenta con presencia en más de 13 países en tres continentes y ha desarrollado proyectos tecnológicos para algunas de las empresas más grandes del mundo como: Orange, Claro, Movistar, Universal Music, LAN, Manchester United, Tigo y Terra.

\section{Desarrollo de la Investigación}

\section{Características de las organizaciones TI y sus proyectos relevantes}

Los resultados obtenidos de la primera sección del cuestionario, correspondientes a la caracterización de las organizaciones TI seleccionadas para el estudio, y la identificación del proyecto más relevante elaborado en los últimos dos años, se presentan en la Tabla 4

Tabla 4. Empresas TI: Características generales y proyecto más relevante

\begin{tabular}{|c|c|c|c|}
\hline Empresa & $\begin{array}{l}\text { LATAM Airlines } \\
\text { Ecuador }\end{array}$ & $\begin{array}{l}\text { Jarflex - Desarrollo de } \\
\text { Soluciones }\end{array}$ & Grupo Link \\
\hline Cantidad de colaboradores & Más de 1000 & 9 & 49 \\
\hline Número de proyectos realizados en & 20 & 17 & 80 \\
\hline los últimos dos años & & & \\
\hline Proyecto relevante & PRAM anuncios & Directorio EC & $\begin{array}{l}\text { Experiencia } \\
\text { única }\end{array}$ \\
\hline Tipología del proyecto relevante & Software & Software & Software \\
\hline
\end{tabular}

A continuación se describe cada uno de los proyectos relevantes de las organizaciones TI seleccionadas como casos de estudio.

\section{PRAM Anuncios}

Es un aplicativo móvil que permite la reproducción de audios pre-grabados, permitiéndole al Agente de Embarque continuar con sus otras tareas, sin tener que interrumpirlas para realizar los anuncios. Al momento, el aplicativo se encuentra funcionando en los aeropuertos de Guayaquil, Islas Galápagos (Baltra y San Cristóbal), y Cuenca, facilitando el proceso de embarque y logrando uniformidad en los anuncios, lo que se traduce en un impacto positivo en la percepción del pasajero. Este proyecto ha sido seleccionado como base, para que el grupo LATAM unifique su sistema de anuncios a nivel mundial. Adicionalmente, el consorcio QUIPORT, concesionario del aeropuerto de Quito, ha solicitado la asesoría para implementarlo como un modelo para todas las aerolíneas que operan en esa ciudad.

\section{Directorio EC}

Es un portal informativo que permite consultar información del personal de LATAM: Nombres, cargo, contactos telefónicos, e información adicional. Esta información es alimentada directamente desde las bases de datos de SAP y se actualiza todos los días. Desde la implementación de este proyecto, los funcionarios pueden ubicar, con facilidad, a sus compañeros de trabajo para reuniones o consultas. Actualmente, Directorio EC es utilizado de forma periódica por los operarios de LATAM, sin reportarse incidencias o quejas. 


\section{Experiencia Única Mi Claro}

Consistió en la renovación de todos los canales de autoservicios (web site, aplicaciones móviles para Android e iOs, sitio móvil, kioscos de autoservicios, y tienda virtual) de Claro Ecuador, bajo el nombre de la plataforma MI CLARO. A través de estos canales, los clientes de Claro pueden realizar transacciones y administrar sus líneas celulares y otros servicios que ofrece la operadora. Al implementarse el proyecto, el índice de acceso a las plataformas virtuales tuvo un aumento de cerca de 20.000 transacciones por mes, y ser registró una disminución de 80.000 visitas a los centros de atención físicos, optimizando así los costos operativos de la empresa.

\section{Evaluación de la gestión de proyectos relevantes}

Para la evaluación de la gestión de los proyectos relevantes, se consultó a los entrevistados sobre el cumplimiento del alcance, tiempo, presupuesto, estándares de calidad, identificaciónt temprana de riesgos, y la satisfacción de los interesados, utlizando una escala Likert de calificación de cinco puntos, donde 5 es el mejor puntaje. Además, se le consultó si utilizaron alguna buena práctica o metodología de gestión de proyectos. En la Tabla 5 se presentan los resultados de la evaluación.

Tabla 5. Empresas TI: Evaluación de la gestión de proyectos relevantes

\begin{tabular}{|c|c|c|c|c|c|c|c|c|c|}
\hline $\begin{array}{l}\text { Proyecto/ } \\
\text { Empresa }\end{array}$ & 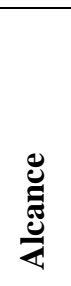 & 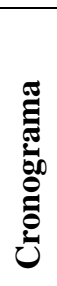 & 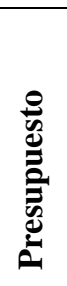 & 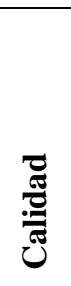 & 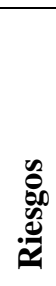 & 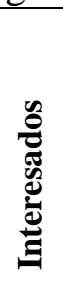 & 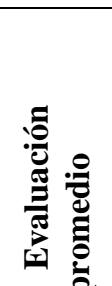 & 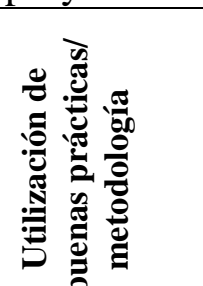 & Sustento de la evaluación \\
\hline \begin{tabular}{l}
\multicolumn{1}{c}{ PRAM } \\
Anuncios/ \\
LATAM \\
Ecuador
\end{tabular} & 5 & 4 & 4 & 5 & 4 & 4 & 4,3 & \begin{tabular}{l}
\multicolumn{1}{c}{ PMI ${ }^{\circledR} /$} \\
Metodología \\
Waterfall
\end{tabular} & $\begin{array}{l}\text { El producto final cumplió con los } \\
\text { requisitos solicitados y los } \\
\text { estándares de calidad esperados por } \\
\text { el cliente, que comprendió la } \\
\text { reproducción de la aplicación en } \\
\text { distintos tipos de dispositivos con } \\
\text { sistemas operativo Android, así } \\
\text { como una fácil inducción a usuarios } \\
\text { nuevos, para lo cual se realizaron } \\
\text { revisiones durante las etapas previas } \\
\text { a desarrollo (documentación y } \\
\text { propuestas gráficas). Se } \\
\text { identificaron riesgos, como la } \\
\text { solicitud de nuevos escenarios de } \\
\text { audio no contemplados en el alcance, } \\
\text { y que estos no fuesen soportados por } \\
\text { el tipo de desarrollo utilizado. El } \\
\text { tiempo y presupuesto no tuvieron } \\
\text { afectaciones notables. }\end{array}$ \\
\hline
\end{tabular}




\begin{tabular}{|c|c|c|c|c|c|c|c|c|c|}
\hline $\begin{array}{r}\text { Directorio } \\
\text { EC/ Jarflex }\end{array}$ & 2 & 4 & 3 & 3 & 3 & 3 & 3,0 & Ninguna & $\begin{array}{l}\text { El proyecto fue dimensionado para } \\
3 \text { meses, sin embargo su liberación } \\
\text { se realizó en } 6 \text { meses con un costo } \\
\text { final de US\$ } 2500 \text {, cuando se } \\
\text { propuso inicialmente US\$ 990. No } \\
\text { se identificaron riesgos de forma } \\
\text { temprana, ni se contemplaron } \\
\text { escenarios y restricciones en la } \\
\text { documentación entregada, lo cual } \\
\text { causó inconformidad en los usuarios. } \\
\text { Su lección aprendida es contemplar } \\
\text { el uso de buenas prácticas o de una } \\
\text { metodología en gestión de proyectos } \\
\text { para cumplir con los requerimientos } \\
\text { de alcance, presupuesto y tiempo. }\end{array}$ \\
\hline
\end{tabular}

\section{Proyecto/ \\ Empresa}

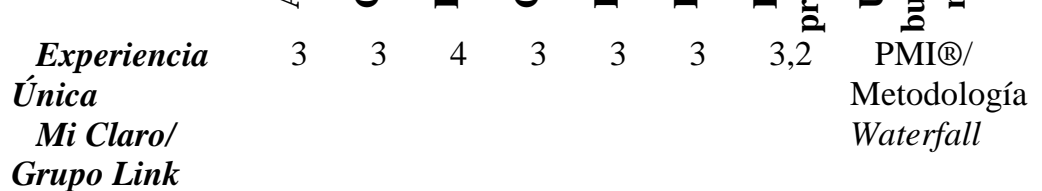

\section{Sustento de la evaluación}

El proyecto fue dimensionado para una duración de 10 meses, aunque el cliente solicitó su liberación en 7 meses. Tras varias fases de pruebas y correcciones, el proyecto culminó en 10 meses y con un costo final de US\$ 60.000, cuando el presupuesto propuesto fue de US\$ 80.000 . Debido a la magnitud del alcance, no se pudieron llevar a cabo revisiones exhaustivas, y se dejaron de lado algunas funcionalidades. No se identificaron tempranamente los riesgos, tales como los escenarios, en casos de uso y tipos de usuarios, para quienes debían habilitarse servicios específicos, según su plan. $\mathrm{Su}$ lección aprendida es mejorar su desempeño en la etapa de levantamiento de información para determinar el alcance y tiempo del proyecto apropiadamente.

Elaborado por: Las autoras. 


\section{Comentarios Finales}

Dos de las tres organizaciones TI participantes del estudio, utilizaron las buenas prácticas del PMI®, y particularmente la metodología waterfall (cascada), que se caracteriza por su simpleza, progresión secuencial y estructurada del trabajo, su orientación a procesos altamente formales, el manejo de mucha documentación, y el planteamiento inicial de requerimientos (alcance), presupuesto y tiempo, con la debilidad de que hay inflexibilidad en los cambios de requerimientos (Awad, 2005). Sin embargo, al momento de evaluar la gestión de proyectos relevantes, sus calificaciones promedio difieren, LATAM Ecuador es notablemente superior a Grupo Link, siendo evidente que una gestión de proyectos exitosa no solo depende de aplicar una buena práctica, sino que también se requiere de un Director de Proyectos, que defina la metodología más apropiada para alcanzar los objetivos tal como lo señalaron Ilies, Crisan, y Muresan (2010). Jarflex y Grupo Link, tuvieron problemas similares en sus proyectos, tales como: incumplimiento de tiempo de entrega, deficiente levantamiento de requerimientos, lo que afectó al alcance del proyecto, y por ende generó insatisfacción de los interesados, y la no identificación temprana de riesgos, por lo tanto, no notaron las señales tempranas de alerta de fracaso usuales en los proyectos TI (Kappelman, McKeeman, y Zhang, 2006). Estos resultados dan una pista del bajo grado de madurez de las organizaciones TI ecuatorianas en la gestión de sus proyectos, ya que además de que una de las organizaciones no utilizó buenas prácticas, otra aun usándolas, no culminó su proyecto con éxito, y fue evidente que con excepción de las buenas prácticas del PMI ${ }^{\circledR}$, los otros estándares internacionales y directrices relevantes para la gestión de proyectos (Drob y Zichil, 2013) son desconocidos.

Este estudio tiene limitaciones: a) Es una investigación exploratoria, al solamente haber considerado tres organizaciones (de diferente tamaño) como casos de estudio, b) el instrumento aplicado carece de preguntas estructuradas para la obtención de características de los proyectos gestionados, y solamente contiene una pregunta dicotómica para indagar el uso de buenas prácticas, y c) es subjetiva la selección del proyecto relevante evaluado, ya que depende de la visión del directivo que llenó el cuestionario. En Ecuador, hay mucho por conocer sobre la gestión de proyectos en organizaciones TI, por lo tanto, cabe proponer futuras investigaciones con alcance descriptivo y/o causal, que impliquen la recolección de un mayor número de casos de estudio, cuyos proyectos puedan ser estratificados por tamaño, tipología, y aplicación de buenas prácticas o metodologías tradicionales como waterfall o nuevas como Agile que son iterativas, de progresión incremental, orientadas a la gente, con poca documentación y con la flexibilidad de adaptar nuevos requerimientos, priorizando la generación de valor al negocio (Awad, 2005). 


\section{Bibliografía}

Awad, M.A. (2005). A comparison between agile and traditional software development methodologies. School of Computer Science and Software Engineering, the University of Western Australia, Perth.

Chemuturi, M., (2013), Mastering IT Project Management: Best Practices, Tools and Techniques, Florida, Estados Unidos: J. Ross Publishing.

Drob, C., y Zichil, V. (2013). Overview regarding the main guidelines, standards and methodologies used in Project Management. Journal of Engineering Studies and Research, 19, (3), 26-31.

Escandón, D.MC. (Septiembre/2005). La gestión de proyectos: Un panorama conceptual. Revista Académica e Institucional de la U.C.P.B, (72), 29-51.

Fuster, H., Hormigo, I., Joana, J., y Rodríguez, J., (2011), Fundamentos de sistemas de información, Barcelona, España: Eureca Media, SL.

Haugan, G.T., (2002), Effective WBS, Vienna, Austria: Management Concepts.

Ilies, L., Crisan, E., y Muresan, I.N. (2010). Best Practices in Project Management. Review of International Comparative Management, 11, (1), 43-51.

International Project Management Association. (Marzo de 2017). About IPMA, disponible en: http://www.ipma.world/

International Organization for Standardization. (Marzo de 2017). All about ISO, disponible en: https://www.iso.org/home.html

Kappelman, L.A., McKeeman, R., y Zhang, L. (2006). Early warnings signs of IT Project failure: The dominant dozen. Information Systems Management, 23, (4), 31-36.

PRINCE2 (Marzo de 2017). About, disponible en: https://www.prince2.com/usa

Project Management Association of Japan. (Marzo de 2017). About PMAJ, disponible en: http://www.pmaj.or.jp/ENG/

Project Management Institute, (2013), Fundamentos para la Dirección de Proyectos (Guía del PMBOK®), Pensilvania, Estados Unidos: Project Management Institute Inc.

Salinas Duarte, A.E. (2007). Obstáculos en la gestión de proyectos en Tecnologías de Información y Comunicación - TICs y posibles soluciones. Artículo de especialización, Universidad Pontificia Bolivariana, Colombia.

Stretton, A. (2007). A Short History of Modern Project Management. PM World Today, IX, (X), 


\section{$1-18$.}

The Standish Group International, (2013), Chaos Manifesto 2013, disponible en: https://www.versionone.com/assets/img/files/CHAOSManifesto2013.pdf.

Vilar, B. (2013). Programación de proyectos con múltiples calendarios. Tesis de maestría, Universidad Politécnica de Valencia, España.

Weaver, P. (2012). Henry L Gantt, 1861 - 1919 Debunking the myths, a retrospective view of his work. PM World Journal, I, (V), 2-10.

Zapata, A., Murillo, G., y Martínez, J., (2006), Organización y Management, Cali, Colombia: Editorial Universidad del Valle. 